NASA Technical Memorandum 88955

\title{
Euler Analysis of the Three Dimensional Flow Field of a High-Speed Propeller: Boundary Condition Effects
}

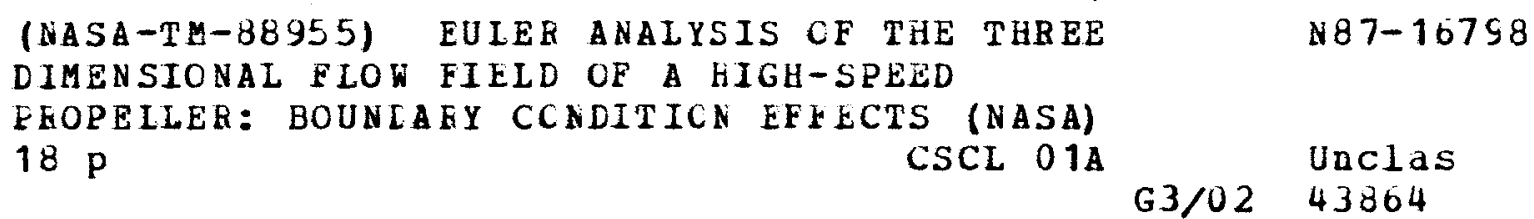

M. Nallasamy

Sverdrup Technology, Inc.

Lewis Research Center

Cleveland, Ohio

and

B.J. Clark and J.F. Groeneweg

Lewis Research Center

Cleveland, Ohio

Prepared for the

32nd International Gas Turbine Conference and Exhibition sponsored by the American Society of Mechanical Engineers Anaheim, California, May 31-June 4, 1987

\section{Nhs}


EULER ANALYSIS OF THE THREF DIMENSIONAL FLOW FIELD OF A HIGH-SPEED PROPELLER: BOUNDARY CONDITION EFFECTS

\author{
M. Nallasamy \\ Sverdrup Technology, Inc. \\ Lewis Research Center \\ Cleveland, Ohio 44135 \\ and \\ B.J. Clark and J.F. Groeneweg \\ National Aeronautics and Space Administration \\ Lewis Research Center \\ Cleveland, Ohio 44135
}

SUMMARY

This paper presents the results of an investigation of the effects of far field boundary conditions on the solution of the three dimensional Euler equations governing the flow field of a high speed single rotation propeller. The results show that the solu-

of tions obtained with the nonreflecting boundary condi-

$\stackrel{m}{p}$ tions are in good agreement with experimental data.

$\dot{\omega}$ The specification of nonreflecting boundary conditions

is effective in reducing the dependence of the solution on the location of the far field boundary. Details of the flow field within the blade passage and the tip vortex are presented. The dependence of the computed power coefficient on the blade setting angle is examined.

\section{NOMENCLATURE}

$\bar{A}$

$C^{+}$

$\mathrm{C}^{-}$

$\mathrm{C}_{\mathrm{p}}$

$\mathrm{C}_{\mathrm{Th}}^{\mathrm{p}}$

c

$c_{p} \quad$ specific heat at constant pressure

0 blade(tip) diameter

$\mathrm{dC}_{\mathrm{Th}} / \mathrm{dx}$ elemental thrust coefficient

$d c_{p} / d x$ elemental power coefficient

E

specific internal energy

$\mathrm{H}$ specific stagnation enthalpy

$\bar{i}$ unit vector

$J$ advance ratio

M Mach number

$\mathrm{n}$ rotational speed, revolutions per sec

p power

$\begin{array}{ll}p & \text { static pressure } \\ R & \text { gas constant } \\ R_{t} & \text { blade (tip) radius } \\ T & \text { static temperature } \\ T h & \text { thrust } \\ t & \text { time } \\ \bar{V} & \text { velocity vector } \\ V_{01} & \text { volume of element } \\ X & \text { fractional radius } \\ z, r, \theta & \text { cylindrical coordinates } \\ B_{3 / 4} & \text { blade angle at } 0.75 \text { blade radius } \\ r & \text { ratio of specific heats } \\ \Delta() & \text { change in a property in a time step } \\ \rho & \text { fluid density } \\ \Omega & \text { rotational speed } \\ \text { Subscripts } & \\ e & \text { extrapolated value } \\ Z, r, \theta & \text { in coordinate directions } \\ \infty & \text { at free stream condition } \\ \text { INTROoUCTION }\end{array}$

Advanced high speed propellers with transonic helical tip Mach numbers are being developed for fue 1 efficient passenger aircraft to operate in the speed range of the current turbofan powered aircraft. High speed propellers incorporate many features which are quite different from low speed ones. Advanced propellers employ eight or ten highly loaded, low aspect ratio, thin and highly swept blades (Fig. 1) to obtain high propulsive efficiency and to reduce noise radiation. To optimize the design of high speed propellers in terms of the aerodynamic and aeroacoustic considerations, a knowledge of the complex flow field is 
essential. Experimental, analytical and numerical approaches are being developed to define and analyze the flow field of an advanced high speed propeller (Mikkelson et al. 1985).

The design procedure for advanced high speed propellers employs the Goldstein type lifting line analysis modified to account for blade sweep, spanwise variation of blade loading and blade-nacelle interaction (Rohrbach et al. 1982). For a detailed analysis and understanding of the high speed propeller flow field, lifting surface analyses are used. The lifting surface analysis used in a full numerical approach to the understanding of the propeller flow field is either a potential flow analysis or an Euler analysis. The potential flow analysis solves the transonic potential equation and requires the specification of wake location. The potential flow solution has been extensively used in aerodynamic analysis. The assumption of potential flow is, however, not strictly correct when shock waves are present. A correct description of the inviscid transonic flow can be be obtained by solving the three dimensional Euler equations. These equations allow entropy rise through the shock waves while conserving mass, momentum and energy. The Fuler formulation provides for the treatment of rotational flows including vortices and strong shock effects. It has been reported that the solution of Euler equations has produced a reasonable description of leading edge vortex and vortex wake behavior (Rizzetta and Shang 1986, Grinstein, et al. 1986). The solutions of three dimensional Euler equations have been found very useful in analyzing complex flows in turbomachines. Recently, they have also been employed to describe the flow fields of high speed single rotation (Barton et al. 1985, Clark and Scott 1986) and counter rotation (Celestina et al. 1986) propellers.

The time dependent Euler equations admit an infinite number of solutions, each one defined by a set of initial and boundary conditions. Initial conditions remaining the same, any change in the boundary condition will produce a new solution. The specification of the boundary conditions for the time dependent Euler equations governing the flow in an infinite domain, such as that of a propeller, needs careful consideration. The numerical solution of the differential equations governing the propeller flow field can be carried out in one of three ways:

(1) Transform the infinite domain to a finite domain and specify the free stream conditions at the far field boundary.

(2) Place the far field boundary at a distance sufficiently far away from the solid surface and specify the free stream conditions on this boundary. or

(3) Place the boundary at a finite distance but relatively close to the region of interest, to keep the number of grid points and hence the computing time within limits. The boundary condition on this boundary then should be modified.

option three is desirable in many respects particularly in terms of grid resolution and accuracy of the solution in the region of interest. However, boundary condition modification becomes complicated for threedimensional flows. Simplified nonreflecting boundary conditions are often employed.

The aim of this paper is to study the sensitivity of the three dimensional Euler predictions to far field boundary conditions. The present investigation considers the application of a simplified nonreflecting far field boundary condition and the effect of the location of the far field boundary in the Euler analysis of a propeller flow field. The solution obtained with the simplified far field boundary condition is found to be in good agreement with experimental data and published Euler analyses. The specification of a nonreflecting boundary condition is shown to be effective in reducing the dependence of the solution on the location of the far field boundary.

Numerical Treatment of Far Field Boundary Conditions Severat investigators have studied the effect of far field boundary conditions on the solution of Euler equations. The excellent study of boundary conditions for supersonic flows by Abbett (1973) clearly pointed out that the characteristic type schemes at boundaries tended to result in optimum accuracy. Approaches developed to reduce the influence of the location of the far field boundary and the boundary condition specified there on the solution of transonic flows include:

(1) A far field boundary condition based on an expansion that was asymptotic in distance from an arbitrary origin, developed by Thomas and Salas (1986) and Bayliss and Turkel (1980).

(2) Construction of a pseudo-differential operator which exactly annihilates the outgoing waves developed by Enqquist and Mazda (1981).

(3) Rudi and Strikwerda's (1980) boundary condition in which the pressure at the outflow section is effectively relaxed to its correct value, using an arbitrary narameter.

(4) Characteristic variable/Riemann invariant specification at the boundary developed by Morretti (1980), Jameson and Baker (1983) and others (for example Celestina et al. 1986).

Kwak (1980) tested several of these boundary conditions on the time dependent small disturbance equation for the flow over an airfoil and came to the conclusion that the characteristic condition is the most effective one in reducing the size of the computational domain and in accelerating converqence. The study of Berry (1984) on the effect of boundary conditions on the solution of (2-D) Euler equations for the flow over an airfoil, showed that the characteristic variable specification at the far field boundary produced the highest rate of convergence. An excellent description of the basis and the formulation of the characteristic boundary conditions for one dimensional flow and extensions to multi-dimensional flows are given by Morretti (1980). To reduce the complexity and effort involved in the treatment of the far field boundary for three-dimensional flows, often it is treated by employing a local one-dimensional approximation and specifying characteristic variables at the boundary (Celestina et al. 1986, Jameson and Baker 1983, Berry 1984). Such a boundary condition is perfectly nonreflecting to waves in one dimension and to waves at normal incidence in two dimensions (Hearstrom 1979). The ideal boundary treatment should take into account all outgoing waves and assure transmission of all waves. Such a technique is described by Erdos et al. (1977). Their technique is, however, too involved and time consuming and is not employed in practice. In this paper, we shall be concerned only with a local one-dimensional approximation technique (item 4 above).

\section{MOTIVATION}

Clark and Scott (1986) employed Denton's (1980) time marching method, which is a finite-volume method developed for turbomachinery flow analysis, to solve the flow field of a high-speed propeller. They modified Denton's combuter program to acconmodate the $f a r$ field boundary of the propeller flow field. They 
specified the far field boundary at a distance of two blade radii from the axis of the propeller and implemented the slip wall boundary conditions there. It has been argued that such a wall boundary specification for a propeller flow in an infinite domain, could produce significant errors in the computed blade pressure distributions which are the input needed for acoustic computations. The importance of an accurate description of the blade pressure distributions has motivated the present study of the effect of far field boundary conditions on the Euler solution of the propeller flow field. In this paper we consider three different far field boundary conditions. They are the free stream condition, solid wall boundary condition and a nonreflecting boundary condition. These conditions are incorporated into the Denton computer program and the solutions are compared.

The experimental data on the high-speed propeller flow field is very limited. Use of the data is difficult because of the uncertainties in the dynamic blade shape. In this paper, the sensitivity of the numerical solution to the blade-setting angle is studied. In addition to the far field boundary condition, the study examines the effect of location of the far field boundary and nonreflecting boundary conditions at the inlet/ exit sections.

\section{Governing Equations}

The governing equations describing the inviscid flow field of the propeller are the three-dimensional Euler equations, expressing the conservation of mass, momenta, and energy. It is convenient to work with absolute quantities, writing the equations in a coordinate frame rotating with the blade, so that centrifugal and coriolis forces $c$ an be represented as body forces acting on each element. The equations are written in the form (Denton 1980):

continuity

$$
V_{01} \Delta \rho=\Delta t \sum \rho \nabla \cdot \bar{A}+\Omega \frac{\partial \rho}{\partial \theta} \Delta t V_{01}
$$

axial momentum

$$
\text { Vol } \begin{aligned}
\Delta\left(\rho V_{Z}\right)=\Delta t \sum\left(P \bar{i}_{Z} \cdot A\right. & \left.+\rho V_{Z} \nabla \cdot \bar{A}\right) \\
& +\Omega \frac{\partial}{\partial \theta}\left(\rho V_{Z}\right) \Delta t V_{01}
\end{aligned}
$$

radial momentum

$$
\text { Vol } \begin{aligned}
\Delta\left(\rho V_{r}\right)=\Delta t \sum\left(\rho \bar{i}_{r} \cdot \bar{A}\right. & \left.+\rho V_{r} \nabla \cdot \bar{A}\right) \\
& +\Omega \frac{\partial}{\partial \theta}\left(\rho V_{r}\right) \Delta t V_{01}
\end{aligned}
$$

angular momentum

$$
\begin{aligned}
V_{01} \Delta\left(\rho r V_{\theta}\right)=\Delta t \sum\left(p r \bar{i}_{\theta} \cdot \bar{A}\right. & \left.+\rho r V_{\theta} \bar{V} \cdot \bar{A}\right) \\
& +\Omega \frac{\partial}{\partial \theta}\left(\rho r V_{\theta}\right) \Delta t V_{0 l}
\end{aligned}
$$

energy

$$
\begin{aligned}
& \text { Vol } \Delta(\rho E)=\Delta t \sum(P H \nabla \cdot \bar{A}) \\
& +\Omega \frac{\partial}{\partial \theta}(\rho E) \Delta t V_{0} l
\end{aligned}
$$

These equations are solved in conjunction with the perfect gas relationships:

$$
\begin{gathered}
P=\rho R T \\
H=C_{p} T+\frac{1}{2} v^{2} \\
\rho E=\frac{P}{r-1}+\frac{1}{2} \rho V^{2}
\end{gathered}
$$

Computational Grid

The governing equations are solved in the physical domain on a grid generated algebraically. The flow domain is discretized using three kinds of surfaces. The bladewise surfaces are evenly spaced and are limited by the suction side of one blade and pressure side of the adjacent one (Fig. 2(a)). In the figure the outer boundary is at a distance $2 R_{t}$ from the axis of the spinner. The streamwise surfaces are variably spaced along the spanwise direction and are limited by the spinner/nacelle surface and the outer boundary (Fig. 2(b)). The spanwise (quasi-orthogonal) surfaces are variably spaced along the axial direction depending on the region, leading edge, trailing edge, blade surface, inlet or exit and are limited by the inlet and exit planes. The computational nodes are located at the corners of each volume element defined by the streamwise, bladewise and spanwise surfaces. The fluxes of mass, momenta, and energy through each face are calculated using the averages of the flow properties stored at the corners of that face.

The Numerical Technique

Denton's explicit time marching method employs what is called an opposed difference scheme. The scheme uses upwind differences for fluxes of mass and momenta, and downwind differences for pressure, in the streamwise direction. The derivatives in the circumferential direction are evaluated using central differences. The time marching method uses a time step such that the Courant number is close to unity. The method itself ensures stability by solving the flow equations in the order of continuity - energy - momentum. The procedure is as follows:

(1) For each time step and each control volume the continuity equation is used to find the new density associated with each grid point.

(2) The energy equation is solved to yield new energy using the densities of the previous time step.

(3) The new density is then used along with the velocities and energy from the previous time step to compute new pressures.

(4) The velocities are then updated using the momentum equations with the new pressures and densities.

A variable time step appropriate for each volume element is used to accelerate convergence. The method is of first order accuracy and is modified to achieve greater accuracy by adding a lagged correction factor to correct the upwind pressure to a value close to the true one. The scheme uses no explicit artificial viscosity. Only a smoothing is used after each time step to smooth out any waviness in the circumferential direction. The convergence is judged on the basis of mass conservation and the maximum change in the axial velocity component.

Initial and Boundary Conditions

The Euler equations governing the flow field of the propeller have to be solved in conjunction with 
appropriate initial and boundary conditions of the flow. Since the steady flow solution is of interest here, the initial conditions need be only approximate. The boundary conditions that need to be specified depend on the flow Mach number. The absolute flow entering and leaving the computational domain is assumed subsonic.

The effect of far field boundary condition on the blade pressure distribution is of main concern in the present study. The boundary conditions considered are:

(1) Wall boundary conditions. The wall boundary condition requires that the mass flux through the surface be zero and the velocity normal to the surface be zero. The implementation of this condition is the same as that of Denton (1980) and Clark and Scott (1986). That is, the mass flux through the far field boundary is simply set to zero and the velocity components and density at this boundary are calculated using the changes in values at two points adjacent to the boundary. The pressure at the boundary is computed as for any inner field point.

(2) Free stream boundary condition. The free stream static pressure, velocity components and density are specified at the far field boundary.

(3) A nonreflecting boundary condition based on a local one dimensional flow approximation and specification of the characteristic variable normal to the boundary. Assuming a fixed incoming characteristic $c^{-}$

$$
C^{-}=V_{\infty}-\frac{2 C_{\infty}}{\gamma-1}
$$

The outgoing characteristic $\mathrm{C}^{+}$is extrapolated from the interior of the computational domain

$$
C^{+}=v_{e}+\frac{2 C_{e}}{r-1}
$$

From the Riemann invariants we can write

$$
\begin{aligned}
& v_{r}=\frac{1}{2}\left(C^{+}+C^{-}\right) \\
& c=\frac{r-1}{4}\left(C^{+}-C^{-}\right)
\end{aligned}
$$

where $V_{r}$ and $c$ are the norinal velocity and speed of sound to be specified at the far field boundary. The entropy is extrapolated (from noints inside the computational domain adjacent to : le boundary) or set equal to the free stream value depending on the direction of the radial velocity. The density, energy and pressure are calculated from the speed of sound and entropy. Depending on the direction of the radial velocity, the axial velocity at the boundary point is computed using either the axial velocity of the free stream or the axial velocity at the point adjacent to the boundary inside the comoutational domain. The tangential velocity component is extrapolated from the point adjacent to the boundary.

A periodicity condition is imposed ahead and downstream of the blade from hub to outer boundary and beyond the blade tip in the blade region. On the solid surfaces the mass flux through the surface is set to zero. At the inlet boundary the relative stagnation temperature, pressure and the flow angle are specified. The axial velocity is computed from the characteristic boundary condition. The upstream running characteris$t$ ic $\mathrm{C}^{-}$is extrapolated from the interior of the computational domain. Then using the specified total temperature and $\mathrm{C}^{-}$the total inlet velocity is computed from isentropic relations. The individual velocity components are computed from geometric relations while density and pressure are calculated from isentropic relations. At the downstream boundary the static pressure is specified at the far field boundary. The radial variation of the static pressure satisfying the radial equilibrium is computed from

$$
\frac{\partial p}{\partial r}=\frac{\rho V_{\theta}^{2}}{r}
$$

Here $V_{\theta}$ and $\rho$ are the extrapolated values from the interior of the domain. The downstream running characteristic, $C^{+}$is also extrapolated. The final density and speed of sound are calculated from isentropic relations. The exit axial velocity is computed from the extrapolated $\mathrm{C}^{+}$characteristic.

\section{RESULTS AND DISCUSSION}

Solutions of SR-3 propeller flow field have been obtained with three different far field boundary conditions, with the boundary located at twice the blade radius from the axis of the propeller. This boundary location was chosen primarily to assess the effects of wall boundary condition specified at the same location used by Clark and Scott (1986). The computations have been carried out for the design conditions of the SR-3 propeller, $\mathrm{J}=3.06, M=0.8$, and $B_{3} / 4=61.3^{\circ}$. Results obtained with the three boundary conditions -wall boundary condition, free stream boundary condition and the nonreflecting boundary condition -- are compared with each other and with experimental data where possible. The effect of location of the far field boundary and details of the propeller flow field are also examined. An accurate description of the pressures on the blade surfaces is essential as they are the input needed for acoustic computations. The effects of the far field boundary conditions are shown in the chordwise pressure distributions plotted in $\mathrm{Fig}$. 3. The figure shows blade surface pressures at $21,53,84$, and 100 percent span heights. First, it is observed that there exists an appreciable difference between the solutions obtained with the wall boundary condition and nonreflecting boundary condition. The large difference stems from the pressure rise introduced due to the confining wall boundary. The difference in pressures between nonreflecting and free stream conditions are appreciable only at 84 and 100 percent locations. It is to be noted that only with the nonreflecting boundary condition are the computed solutions in good agreement with experimental data as discussed below.

Figure 4 shows the spanwise variation of the elemental power coefficient, $d c_{p} / d x$ obtained with the three boundary conditions. Also shown for comparison are the experimental data (Mikkelson et al. 1985) and the results of the lifting line analys is of the design condition (Rohrbach et a1. 1982). The curve for the nonreflecting boundary condition shows good agreement with the experimental data. The variation of the elemental power coefficient above 50 percent span height including the location of the neak power coefficient is well predicted. The Euler predictions near the blade root are poor in all cases. The lifting line analysis predicts well near the blade root but the prediction in the region of peak power coefficient is poor.

The spanwise variation of the elemental thrust coefficient, $d C_{T H} / d x$, is shown in Fig. 5. As before 
the curve for the nonreflecting boundary condition shows good overall agreement with experimental data (Jeracki 1986). All of the three Euler predictions and the lifting line analysis significantly overpredict near the blade root region. The present results show a "transition" region in the predicted elemental thrust coefficient curve, which corresponds to the region of blade shape transition. The SR-3 propeller design incorporates airfoil sections of NACA series 16 from tip to 53 percent radius and NACA series 65 circular arc camber lines from 37 percent radius to the root with a transition fairing between. Detailed examination of projected areas of the blade elements gave no indication of a transition. Limited runs made with different grid densities did not alter the shape of the elemental thrust curve. Although the experimental data indicates a change in slope of the curve, it does not show a distinct transition region as in the predictions. It should be noted here that the measurement of the elemental thrust coefficient is not as direct as the power coefficient. The experimental data shown in Fig. 5 have been scaled to match the measured total thrust coefficient.

Another quantity of interest is the flow swirl angle downstream of the blade. Figure 6 shows the swirl angles predicted in the present study along with experimental data and predictions of Barton et al. (1985). (The Euler analysis of Barton et al. (1985) specifies free stream conditions at the far field boundary located at 9 radii from the axis of the propeller.) Use of the nonreflecting boundary condition tends to move the predicted swirl angles closer to the data.

The sensitivity to the choice of far field boundary location is small when the boundary is nonreflecting. Figure 7 shows pressure distributions for three boundary locations, namely, $2 R_{t}, 2.5 R_{t}$, and $3 R_{t}$. Results indicate that a noticeable difference in pressures obtained with the far field boundary located at 2 and $2.5 R_{t}$, occurs at 84 percent span height and at the blade tip. However, the total power coefficient changes by only about 3 percent. The difference between the blade pressures obtained with the far field boundary at $2.5 R_{t}$ and $3 R_{t}$ is negligible indicating that $2.5 R_{t}$ is sufficiently far when the nonreflecting boundary condition is used. Since the total power coefficient change in moving the $f$ ar field boundary from $2 R_{t}$ to $2.5 R_{t}$ is small, comparisons with data are made with the boundary at $2 R_{t}$ as in the paper of Clark and Scott. It may be noted here that the comparison has been carried out with the same number of grid point: in the radial direction. After the implementation of the nonreflecting boundary condition at the far field, incorporation of the nonreflecting boundary conditions at the inlet/exit does not produce any noticeable difference in blade pressure distributions. For one- and two-dimensional flows, implementation of the nonreflecting far field boundary condition has been found to accelerate the convergence rate (Rudi and Strikwerda 1980 , Berry 1984) of the solution to steady state. In the present computations only a 10 to 20 percent reduction in the number of iterations has been noted with the nonreflecting boundary conditions.

We have observed that the solution obtained with the nonreflecting boundary condition is in reasonably good agreement with experimental data. It is then instructive to examine the complete flow field provided by this solution. The contour plots of pressures in the blade passages are shown in Figs. 8 and 9 . The figures show contour plots for two adjacent blade passages to aid understanding of the flow field. Fig. 8 shows pressure contours at the blade root, midspan and blade tip. The contours show the highly threedimensional nature of the flow within blade passages. The blade - nacelle interaction effects are reflected in the contours at the blade root (Fig. 8(a)). The predicted flow is in qualitative agreement with other predictions and and experiments summarized by Mikkelson et al. (1985). The pressure distributions at three axial stations, namely, leading edge, mid chord, and trailing edge are shown Fig. 9. The pressure contours at the trailina edqe (Fig. 9(C)) indicate the propagation of pressure waves from the blade tio as pictured in color graphics by Adamzyck (1986). The mid chord section contours show the nature of the pressure differential existing between the two sides of the biade.

Figure 10 shows the vorticity contours at two axial stations downstream of the trailing edge. of particular interest are the vorticity contours in the tip region. One can observe the movement of the center of the vortex (marked by $\Delta 9$ in Fig. 10(b)) in the direction of rotation of the blades. Such a displacement of the vortex from the blade wake has been observed in laser Doppler measurements of the highspeed propeller flow field (Neumann et al. 1983). It is attributed to the tip vortex roll-up. One can also notice diffusion of vortex with axial distance, due to numerical diffusion. However, the fact that Euler solutions do provide a reasonable description of the vortex wake and leading edge vortex flows has been observed by many investigators (for example Rizzetta and Shang 1986, Grinstein et al. 1986). Recently, Hanson (1986) estimated the noise produced by the radial forces associated with the tip vortex of a highspeed propeller.

Experimental data on high-speed propellers indicate a strong dependence of the total power coefficient on the blade shape. It is difficult to determine pre-cisely the dynamic blade shape. Here, the sensitivity of the numerical solution to the blade setting anqle is examined. The design biade angle of the SR-3 propeller at $3 / 4$ radius is $61.3^{\circ}$, which untwists to $58.7^{\circ}$, with centrifugal loading at design speed. The computations reported in this paper are with a blade anglo of $58.7^{\circ}$, allowing for the dynanic untwist. The dependence of the computed power coefficient on the blade setting angle is shown in Fig. 11. It is seen that the power coefficient depends strongly on the blade setting angle (or the estimated untwist). Such a dependence has been observed in experiments (Rohrbach et al. 1982) and other Euler analysis (Barton et al. 1985).

\section{CONCLIJSIONS}

The solution of the three-dimensional Euler equations governing the flow field of a high-speed propeller with nonreflecting boundary conditions yields results which are in fairly good aqreement with experimental data. The solution obtained with the wall boundary condition at the far field overpredicts the blade loading in the reqion of the peak by as much as 20 percent. The resulting overprediction in the total power coefficient is about 14 percent. The study also shows that with the specification of the nonreflecting boundary condition, the far field boundary $c$ an be located as close as 2.5 blade radi i from the axis of the propeller without affecting the computed solution.

\section{ACKNOWLEDGMENTS}

The authors wish to acknowledge the helpful discussions with Messrs. M. Celestina and R. Jeracki and Drs. C.J. Miller, L. Bober, and J. Adamczyk during the present investigation. 


\section{REFERENCES}

Abbett, M.J., "Boundary Condition Calculation Procedures for Inviscid Supersonic Flow Fields," Computational Fluid Dynamics Conference, AIAA, New York, 1973, pp. 153-172.

Adamzyck, J.J., private communication, 1986.

Barton, J.M., Yamamoto, 0., and Bober, L.J.,

"Inviscid Analysis of Advanced Turboprop Propeller

Flow Fields," AIAA Paper 85-1263, July 1985.

Bayliss, A. and Turkel, E., "Radiation Boundary

Conditions for Wave Like Equations", Communications

in Pure and Applied Mathematics, Vol. 33, Nov. 1980,

pp. 707-725.

Berry, P.E., "Computation of Unsteady Flow in a

Blade Passage," Unsteady Aerodynamics of Turbomachines

and Propellers, D.S. Whitehead, ed., University of

Cambridge, Cambridge, England, 1984, pp. 191-203.

Celestina, M.L., Mulac, R.A., and Adamzyck, J.J., "A

Numerical Simulation of the Inviscid Flow Through a Counter-Rotating Propeller," NASA TM-87200, 1986.

Clark, B.J. and Scott, J.R., "Coupled Aerodynamic and Acoustical Prediction of Turboprops," NASA

TM-87094, 1986.

Denton, J.D., "Time Marching Methods for

Turbomachinery Flow Calculations," Numerical Methods

in Applied Fluid Dynamics, B. Hunt, ed., Academic

Press, New York, 1980, pp. 473-493.

Engquist, B. and Mazda, A., "Numerical Radiation Boundary Conditions for Unsteady Transonic Flow", Journal of Computational Physics, Vol. 40, No. 1, Mar. 1981, pp. 91-103.

Erdos, J.I., Alzner, E., and Kalben, P., "Computation of Steady and Periodic Two-dimensional Nonlinear Transonic Flows in Fan and Compressor Stages," Transonic Flow Problems in Turbomachinery,

T.C. Adamson and M.F. PTatzer, eds., Hemisphere

Publishing Corp., Washington, 1977, pp. 95-111.

Grinstein, F.F., Oran, E.S., and Boris, J.P.,

"Numerical Simulations of Asymmetric Mixing in Planar Shear Flows", Journal of Fluid Mechanics, Vol. 165, Apr. 1986, pp. 201-220.
Hanson, D.B., "Propeller Noise Caused by Blade Tin Radial Forces," AIAA Paper, 86-1892, July 1986.

Headstrom, G.W., "Nonreflecting Boundary Conditions for Nonlinear Hyperbolic Systems," Journal of Computational Physics, Vol. 30, No. 2 , Feb. 1979, pp. 222-237.

Jameson, A. and Baker, T.J., "Solution of the Euler Equations for Complex Configurations", AIAA Paper, 83-1929, July 1983.

Jeracki, R.J., 1986, unpublished data.

Kwak, D., "Nonreflecting Far Field Boundary

Condition for Unsteady Transonic Flow Computation,"

AIAA Paper, 80-1393, July 1980.

Mikkelson, D.C., Mitchel1, G.R., and Bober, L.,

"Summary of Recent NASA Propeller Research,"

Aerodynamics and Acoustics of Propellers, AGARD

CP-366, AGARD, France, 1985, pp. 12.01-12.24.

Morretti, G., "A Physical Approach to the Numerical Treatment of Boundaries in Gas Dynamics, "Numerical Boundary Condition Procedures, NASA CP-2201, 1980,

pp. 73-95.

Neumann, H.E., Bober, L.J., Serafini, J.S., and

Chang, L.K., "An Analytical and Experimental

Comparison of the Flow Field of an Advanced Swept

Turboprop," AIAA Paper 83-0189, Jan. 1983.

Rizzetta, D.P. and Shang, J.S., "Numerical

Simulation of Leading-Edge Vortex Flows", AIAA

Journal, Vol. 24, No. 2, Feb. 1986, pp. 237-245. Rohrbach, C., Metzger, F.B., Black, D.M., and Ladden, R.M., "Evaluation of Wind Tunnel Performance Testings of an Advanced 45 Swept Eight Bladed Propeller at Mach Numbers from 0.45 to 0.85 ," NASA CR-3505, 1982.

Rudi, O.H. and Strikwerda, J.C., "A Nonreflecting Out Flow Boundary Condition for Subsonic Navier-Stokes Calculations," Journal of Computational Physics, Vol. 36, No. 1, June 1980, pp. 55-70.

Thomas, J.L. and Salas, M.D., "Far-Field Boundary Conditions for Transonic Lifting Solutions to the Euler Equations," AIAA Journal, Vol. 24, No. 7, July 1986, pp. 1074-1080. 


\section{ORIGINAL FAST IS
OF POOR QUALITY}

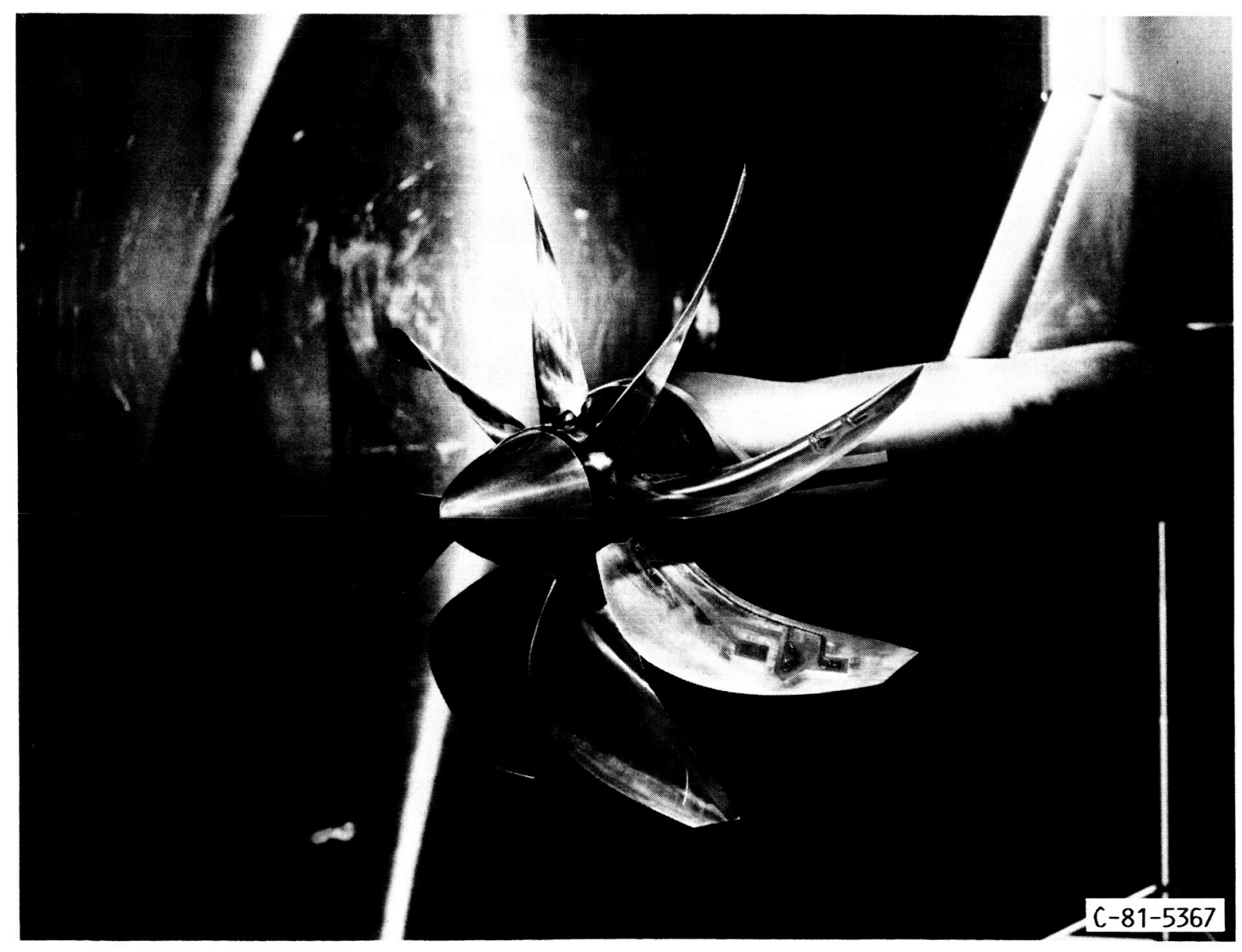

FIGURE 1. - SR-3 PROPELLER. 


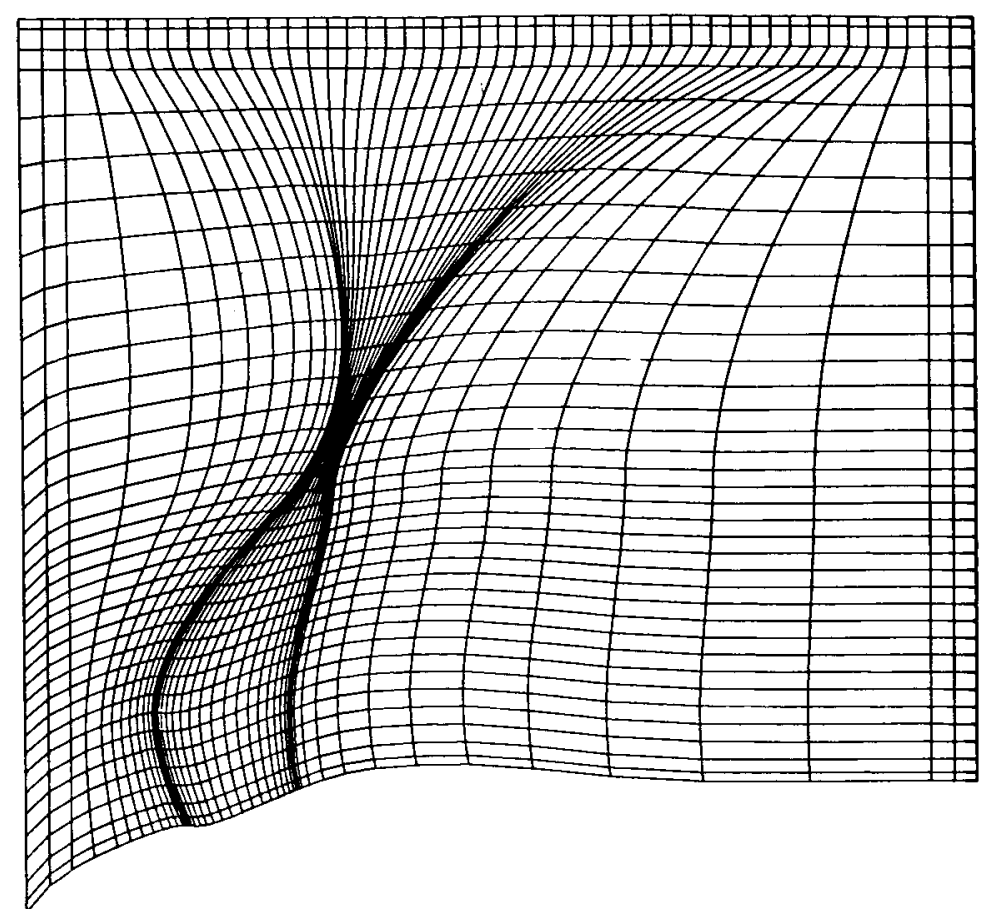

(A) BLADEWISE SURFACE.

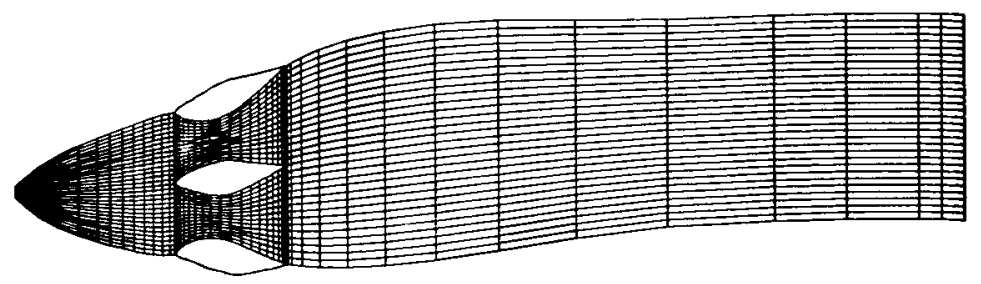

(B) STREAMWISE SURFACE.

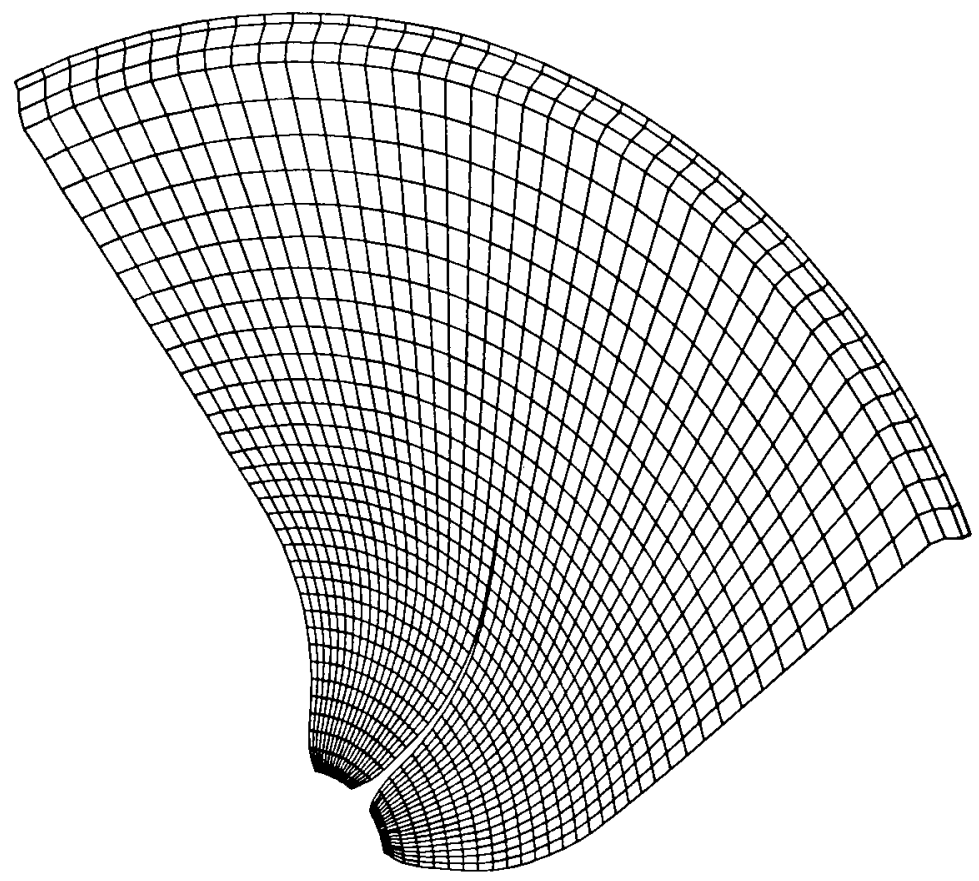

(C) SPANWISE SURFACE.

FIGURE 2. - COMPUTATIONAL GRID. 

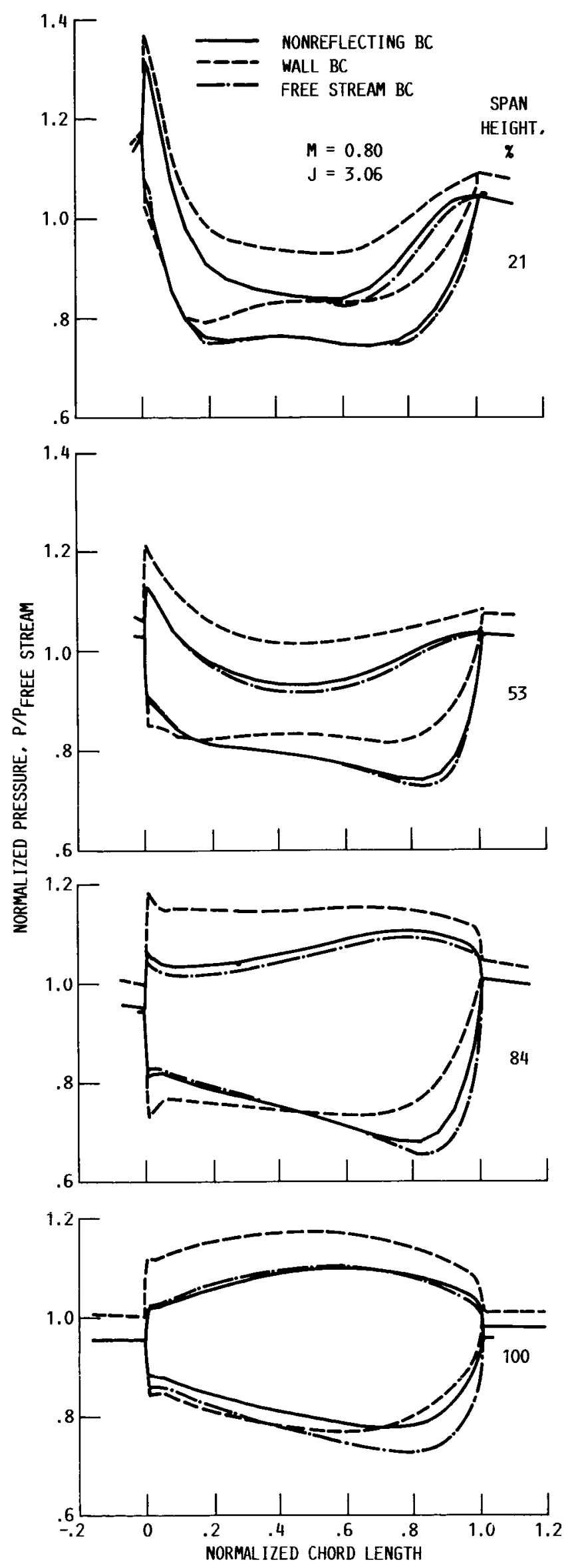

FIGURE 3. - COMPUTED BLADE PRESSURE DISTRIBUTION. 


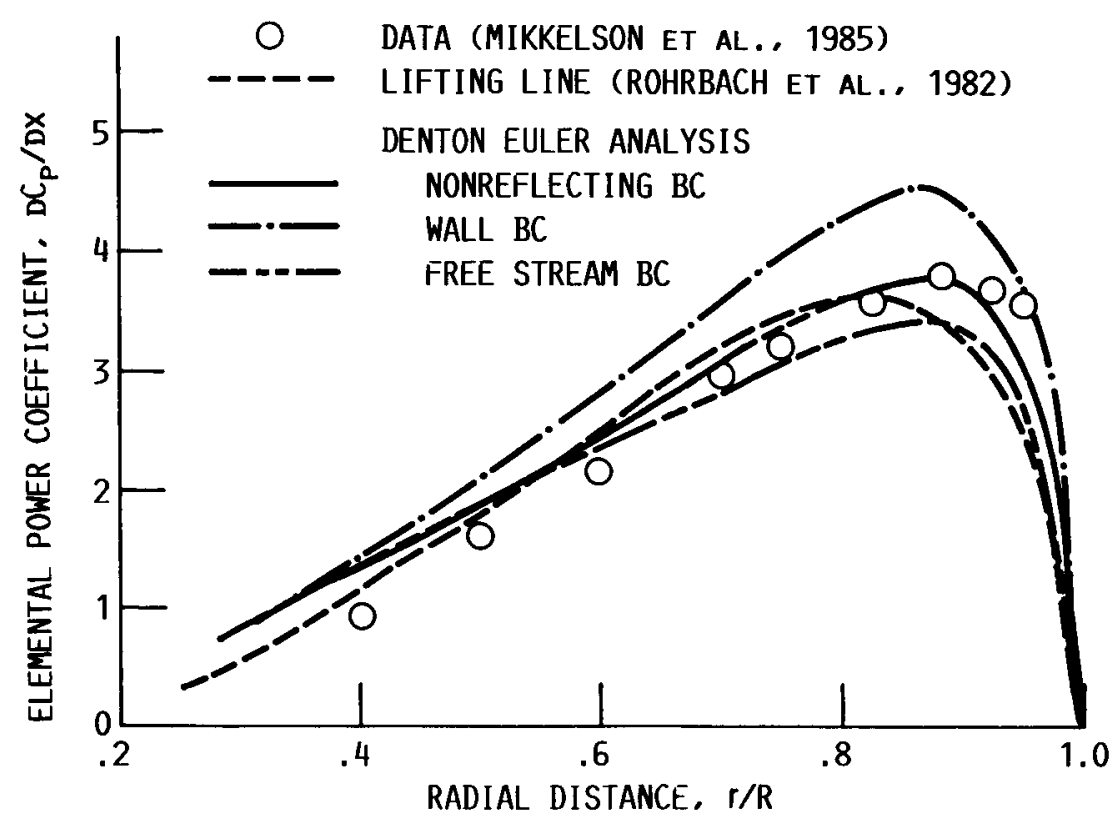

FIGURE 4. - ELEMENTAL POWER COEFFICIENT.

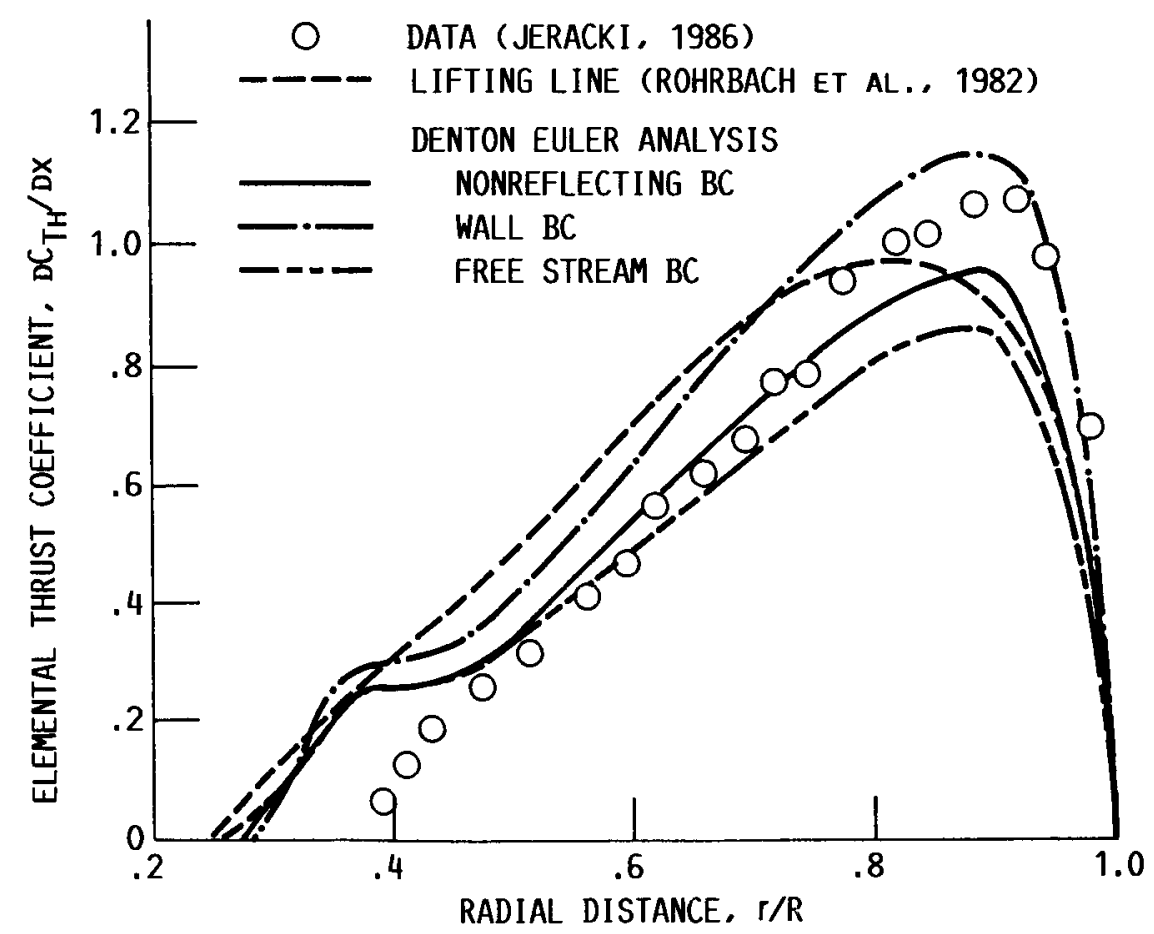

FIGURE 5. - ELEMENTAL THRUST COEFFICIENT. 


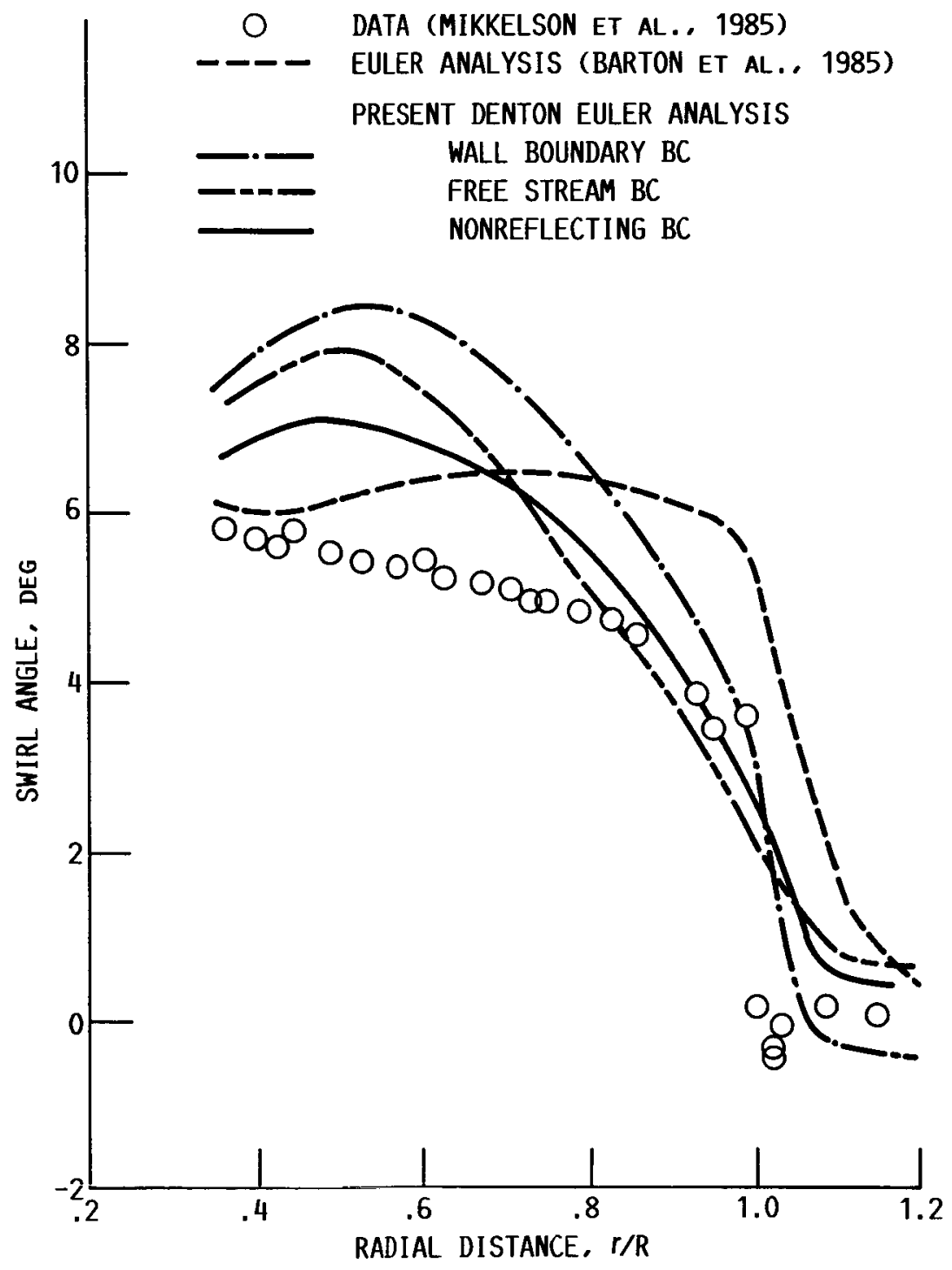

FIGURE 6. - SWIRL ANGLE COMPARISONS AT 0.21 DIAMETER DOWNSTREAM. 

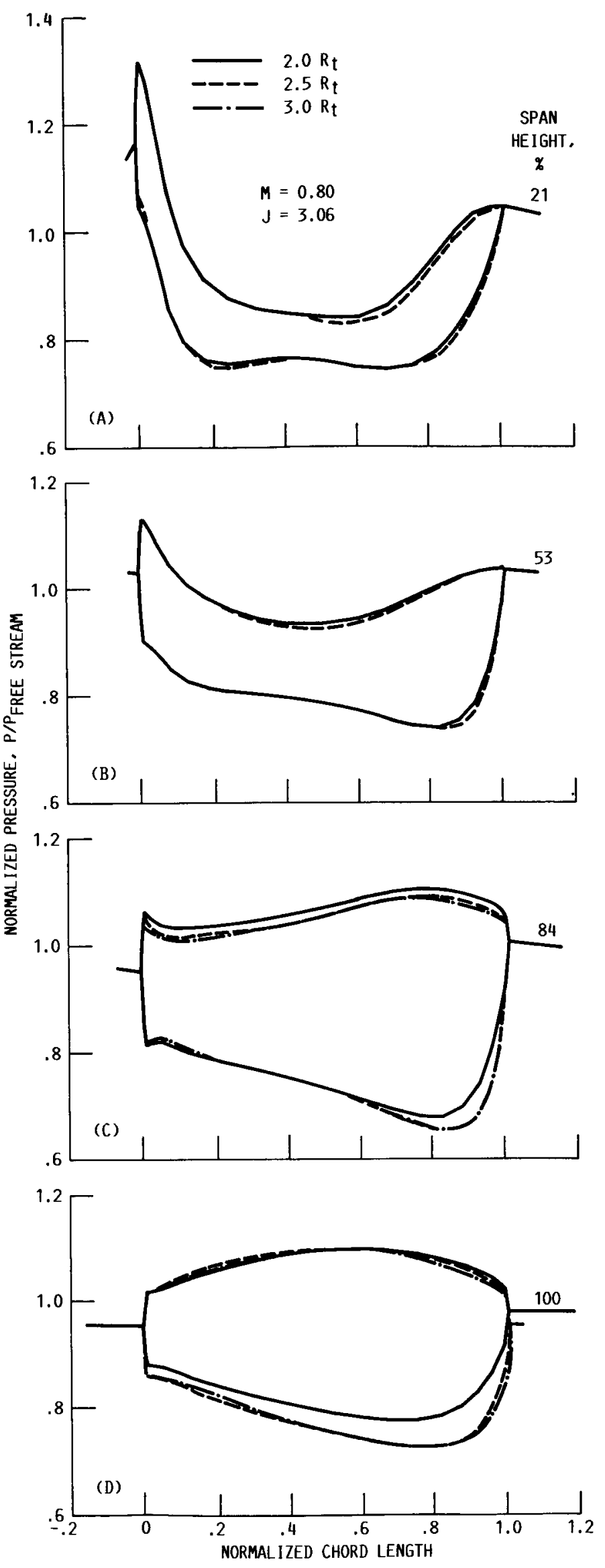

FIGURE 7. - EFFECT OF LOCATION OF FAR FIELD BOUNDARY ON COMPUTED BLADE PRESSURE DISTRIBUTION. 


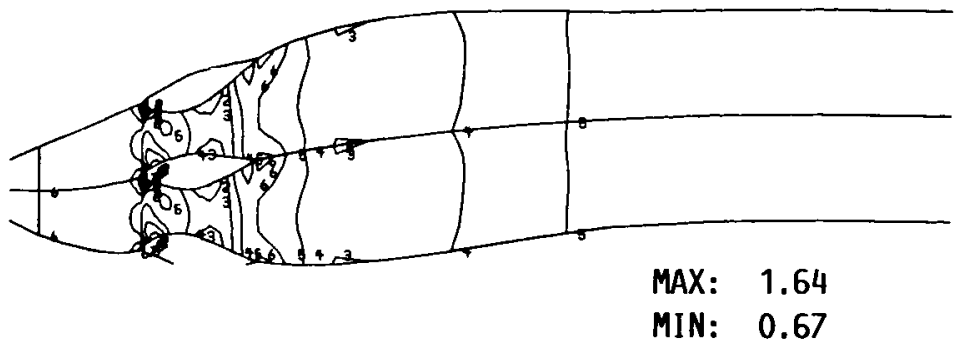

(A) HUB.

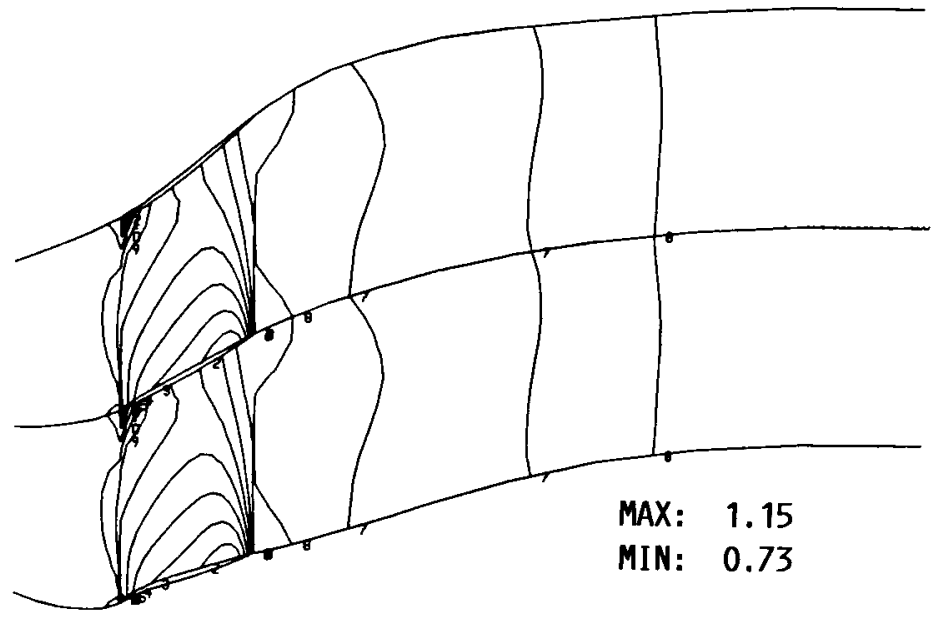

(B) MIDSPAN.

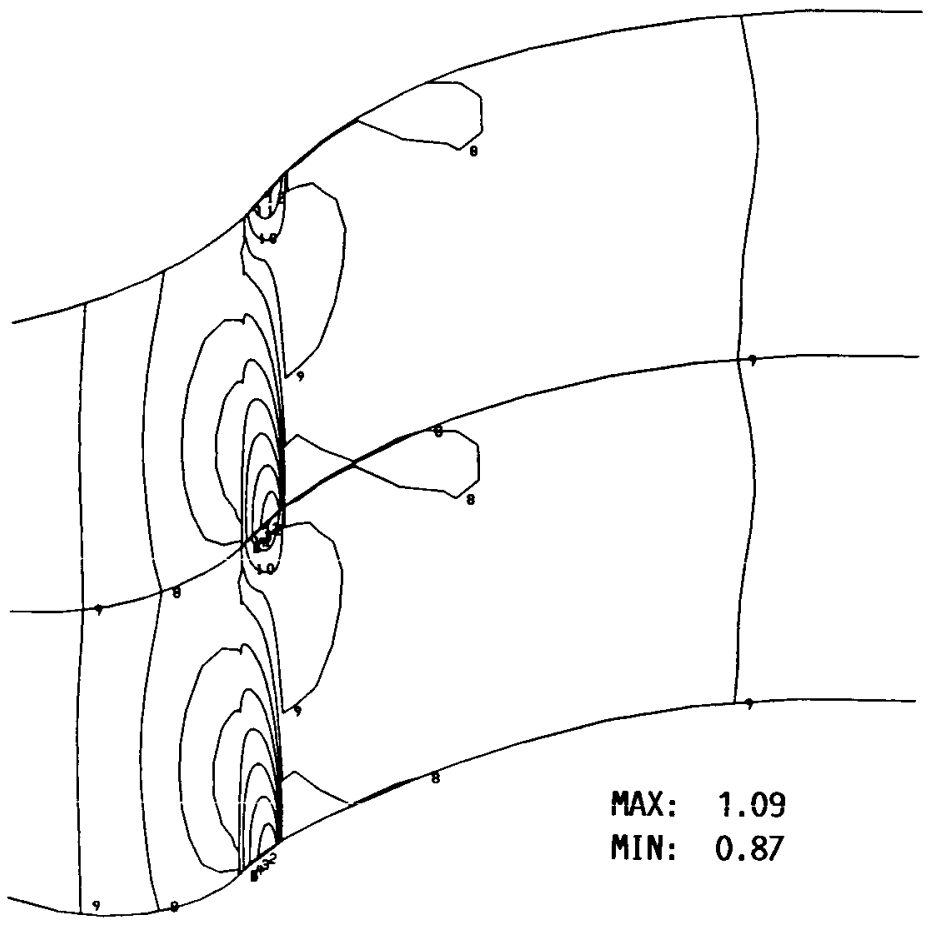

(C) TIP.

FIGURE 8. - COMPUTED PRESSURE DISTRIBUTION ON STREAMWISE SURFACES. $M=0.8, J=3.06$. 


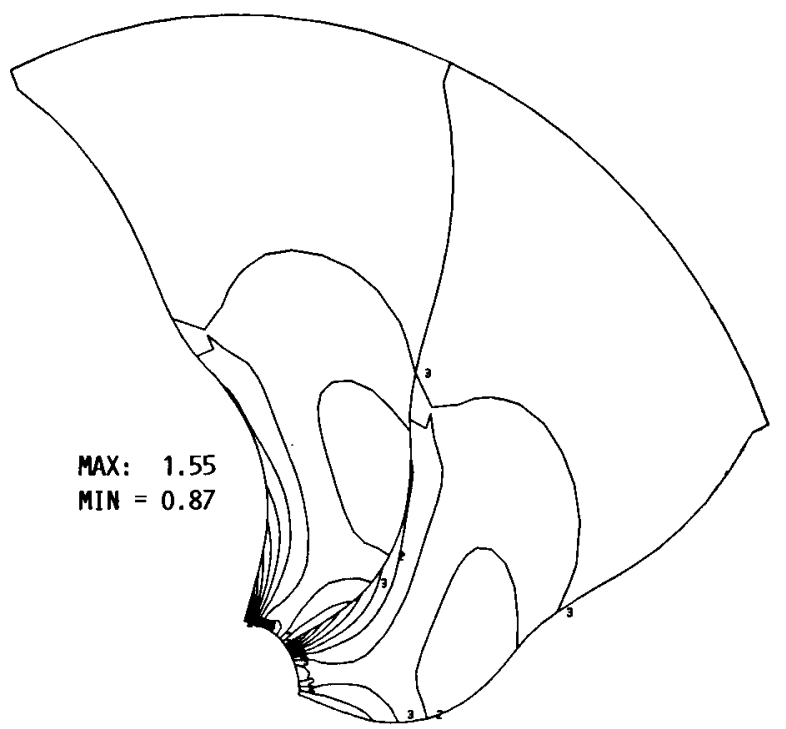

(A) LEADING EDGE.

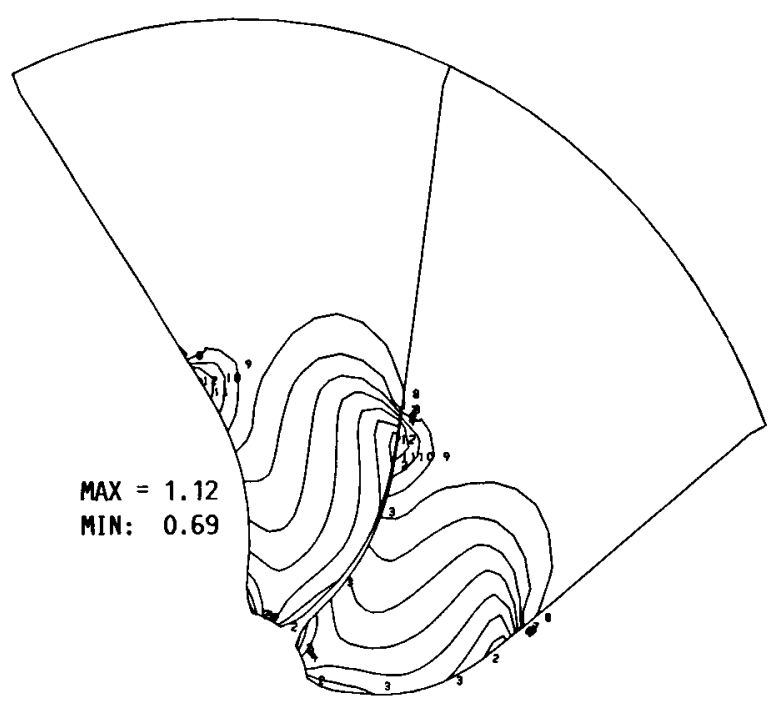

(B) MIDCHORAD.

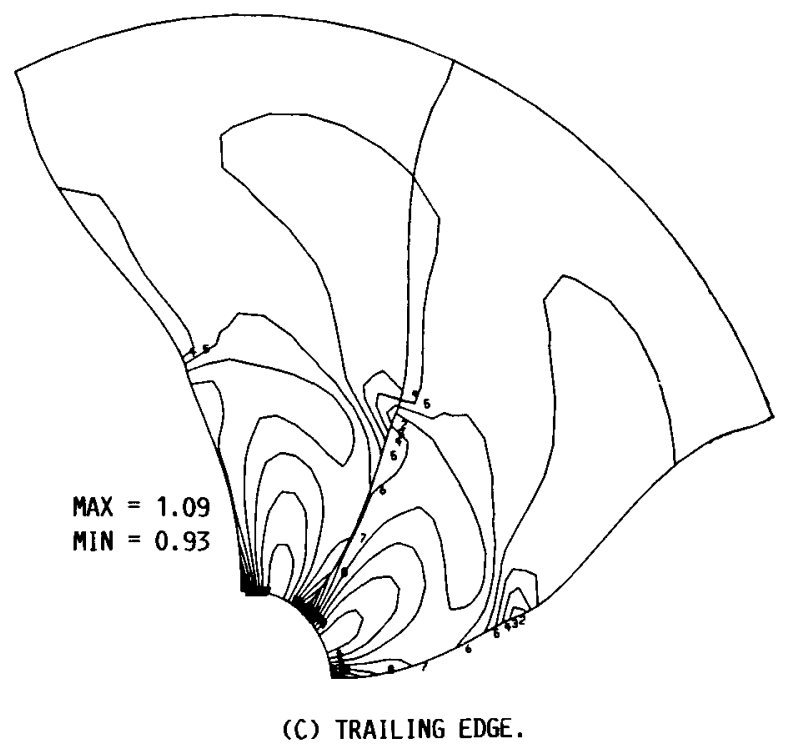

FIGURE 9. - COMPUTED PRESSURE DISTRIBUTION ON SPANWISE SURFACES. $M=0.8, \mathrm{~J}=3.06$. 


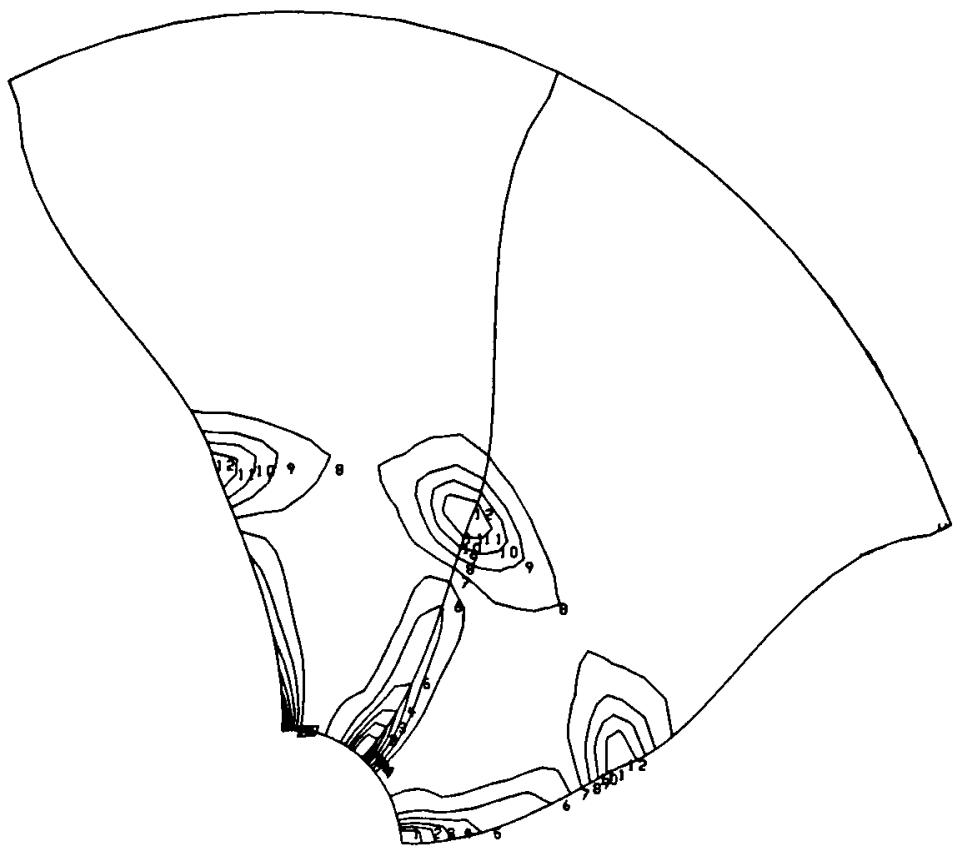

(A) $\omega_{C}=2.25$.

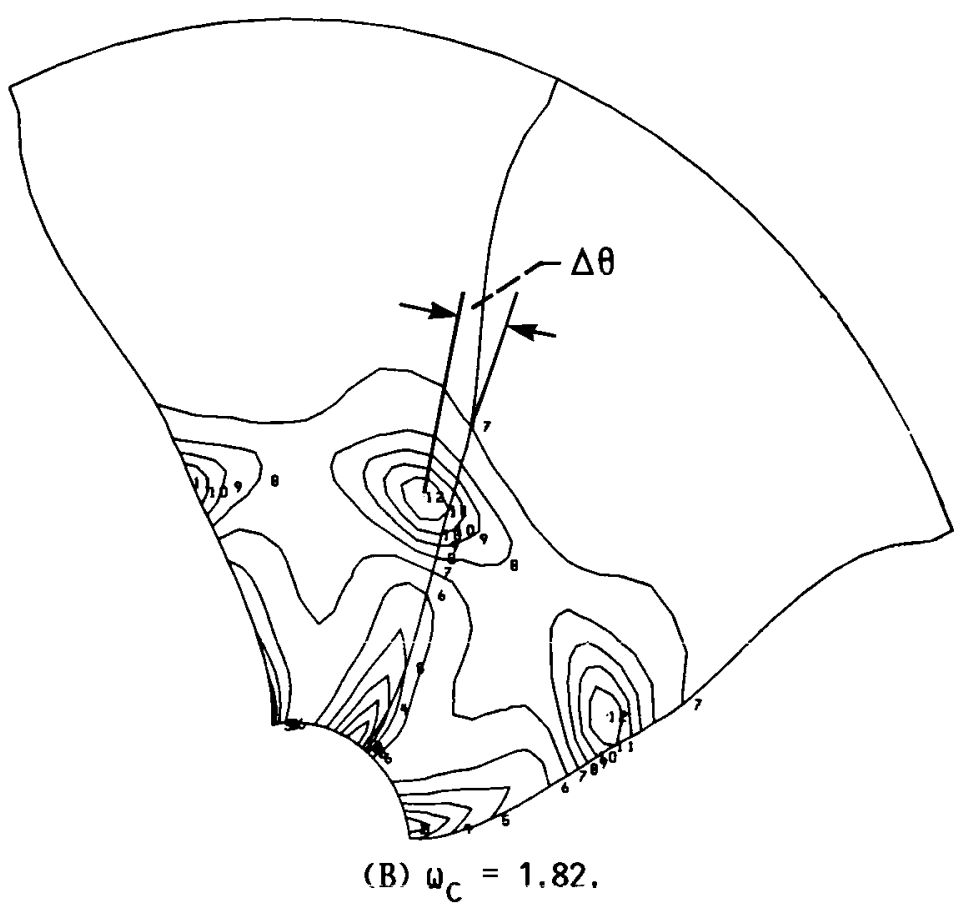

FIGURE 10. - COMPUTED VORTICITY DISTRIBUTIONS.

$\omega_{C}=$ VORTICITY AT THE VORTEX CENTER. 


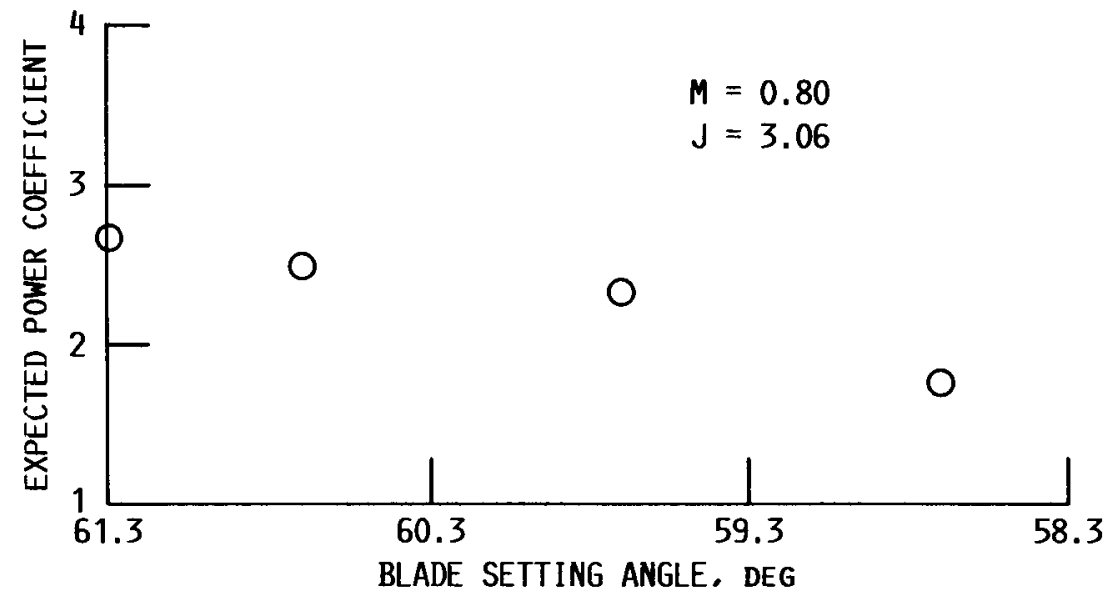

FIGURE 11. - EFFECT OF BLADE SETTING ANGLE. 


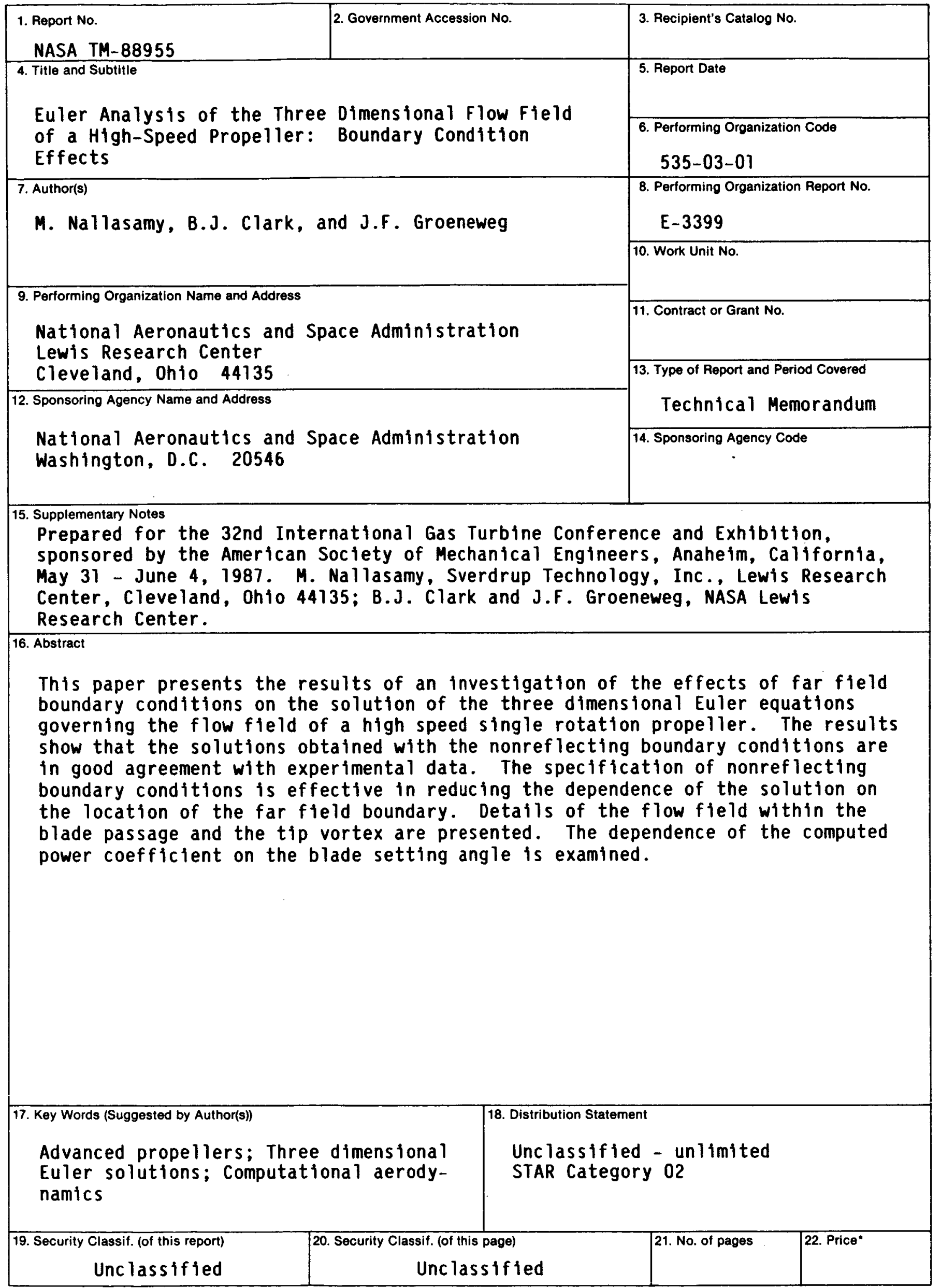

